Mittheilung aus dem I, chemischen Universitätslaboratorium zu Berlin.

Ueber das Hydantoïn und Isomerien bei den Methylhydantoïnen;

von C. Harries und Maurus Weiss.

(Eingelaufen am 20. Mär\% 1903.)

In einer im December des Jahres 1900 erschienenen Mittheilung $\left.{ }^{1}\right)$ haben wir eine bequeme Darstellungsweise des $\mathrm{Hy}$ dantoïns beschrieben. Sie besteht darin, dass man salzsauren Glycocollester zunächst mit Kaliumcyanat umsetzt und darauf den entstandenen Hydantoïnsäureester entweder schmilzt oder mit 25 procentiger Salzsäure eindampft:

$$
\begin{aligned}
& \int_{\mathrm{COOC}_{2} \mathrm{H}_{5}}^{\mathrm{CH}_{2}-\mathrm{NH}_{2} \cdot \mathrm{HCl}}+\mathrm{KOCN}=\mathrm{CO}_{\mathrm{NH} \cdot \mathrm{CH}_{2} \cdot \mathrm{COOC}_{2} \mathrm{H}_{5}}^{\mathrm{NH}_{2}}+\mathrm{KCl} \text {. } \\
& \mathrm{CO}\left\langle\sum_{\mathrm{CH} \cdot \mathrm{CH}_{2} \cdot \mathrm{COOC}_{2} \mathrm{H}_{\overline{3}}}^{\mathrm{NH}_{2}}=\mathrm{CO} \cdot \frac{{ }_{\mathrm{NH}} \cdot \mathrm{CO}}{\mathrm{NH}-\mathrm{CH}_{2}}+\mathrm{C}_{9} \mathrm{H}_{5} \mathrm{OH}\right. \text {. }
\end{aligned}
$$

In dieser Mittheilung wurde der Schmelzpunkt des Hydantoïns etwas höher angegeben $\left(217-220^{\circ}\right)$, als bisher für gewöhnlich beobachtet war und deshalb eine Isomerie vermuthet; inzwischen haben Tafel und Reindla) das Hydantoïn als Product der elektrolytischen Reduction der Parabansäure isolirt und für ihr Präparat diesen höheren Schmelzpunkt ebenfalls gefunden. Wir bemerken deshalb ausdrücklich, dass das aus dem Ester gewonnene Hydantoin in allen seinen Eigenschaften mit dem bisher bekannten Hydantoïn übereinstimmt und dass auch seine Derivate denselben Schmelzpunkt wie die bereits früher gewonnenen anzeigen. Der Schmelzpunkt ist lediglich von der Schnelligkeit des Erhitzens abhängig, da dasselbe hier-

1) Ber. d. deutsch. chem. Ges. 33, 3418 (1900).

2) Ber. d. dentsch. chem. Ges. 34, 3288 (1901). 
bei eine Zersetzung erfährt. Erhitzt man langsam, so findet man den Schmelzpunkt häufig schon bei $214^{\circ}$, während er bei schnellerem Erhitzen bis auf $220^{\circ}$ steigen kann.

Wie mit dem Kaliumcyanat zu Hydantoïnsäure, setzt sich in aualoger Weise das Glyeocollesterchlorbydrat mit Rhodankalium zu Thiohydantoïnsäureester,

$$
\mathrm{Cs}<\begin{aligned}
& \mathrm{NH}_{2} \\
& \mathrm{NH} . \mathrm{CH}_{2} \cdot \mathrm{COO} \cdot \mathrm{C}_{2} \mathrm{H}_{5}
\end{aligned},
$$

um. Ein Versuch, den Thiohydantoïnsäureester durch Schmelzen oder Behandeln mit Salzsäure in das normale Thiohydantoïn:<smiles>ONCN[Ge]</smiles>

umzuwandeln, führte zu keinem positiven Erfolge. Es sei hierbei daran erinnert, dass das von mehreren Forschern ${ }^{3}$ ) auf mannigfaltige Art erhaltene geschwefelte Hydantö̈ı, nach den von Liebermann und Lange ${ }^{4}$ ) angestellten Versuchen, nicht als das oben bezeichnete normale Thiohydantoïn $\mathrm{zu}$ betrachten ist. Vielmebr stellt es sich als Derivat der Imidocarbamidthioessigsäure dar und liefert, entsprechend der Formel:

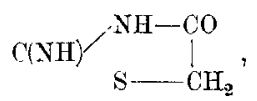

bei der Spaltung Thioglycolsäure.

Für das methylirte Hydantoïn ergiebt sich auf den ersten Blick die Möglichkeit von drei isomeren Monomethylderivaten und zwar:

I.<smiles>CC(C)C(=O)NNC(=O)[O-]</smiles>

II.<smiles>O=[14C]1CCCCC1</smiles>

III.<smiles>CC(=O)CNC(C)=O</smiles>

3) Volhard, diese Annalen 166, 383; Haly, ebenda 168, 133; Ber. d. deutsch, chem. Ges. 8, 1264; 10, 1853; Dixon, Proc. chem. soc. 123, 115; Andreasch, Ber. d. deutsch. chem. Ges. 13, 1422; Monatsh. 8, 424; Claesson, Ber. d. deutsch. chem. Ges. 10, 1349; Niol ati, Gaz. chim. ital. 23, 94.

4) Diese Annalen 207, 132 . 
Das Methylhydantoin I., als das erste Homologe des $\mathrm{Hy}-$ dantoïns betrachtet, führt den Namen ,Lactylharnstoff" und stellt das Anhydrid der zugehörigen Lacturaminsäure:

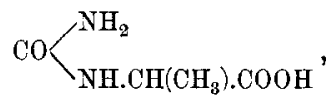

dar. Diese wurde 1873 von Urech ${ }^{5}$ ) durch Verdunsten einer Lösung von Alaninsulfat and Kalinmcyanat dargestellt und durch Schmelzen in Lactylharnstoff übergeführt. Eine von Urech ${ }^{6}$ ) und von $\mathrm{Heintz}{ }^{2}$ ) zu gleicher Zeit aufgefundene Bildungsweise dieses Körpers beruht darauf, dass man Aldehydammoniak mit Cyankalium und Kaliumcyanat in stark schwefelsaurer Lösung längere Zeit stehen lässt und dann bis zur Krystallisation eindampft.

Wir erhielten nach einem der Darstellungsmethode des Hydantoïns analogen Verfahren den Lactylharnstoff aus dem Aetbylester der Lacturaminsäure. Letzteren erhielten wir aus Alanin, indem wir dasselbe mit kochendem Alkohol im Salzsäurestrome esterificirten und den so bereiteten salzsauren Alaninester mit Kaliumcyanat in concentrirter wässriger Lösung bei $50^{\circ}$ condensirten. Die Bildung des Esters vollzieht sich beinahe mit derselben Leichtigkeit, wie die seines niederen Homologen, doch in minder guter Ausbente. Die Alkoholabspaltung aus dem Ester wird ebenfalls durch Kochen mit 25 procentiger Salzsäure bewirkt.

Die Constitution des unter II. angeführten Methylhydantoïns steht ganz zweifellos fest. Es beansprucht insofern ein grösseres Interesse, als es von Schultzen ${ }^{8}$ ) nach Fütterung von Sarkosin im Harn von Hunden aufgefunden wurde. Seine Constitution ergiebt sich unmittelbar aus seinen Darstellungsweisen, von denen hauptsächlich drei erwähnenswerth sind.

5) Diese Anmalen 165, 199.

6) Ber. d. deutsch. chem. Ges. 6, 1113.

¡) Diese Annalen 169, 125.

8) Ber. d. deutsch, chem. Ges. 5, 579. 
Neubauer gewann es, indem er Kreatinin ${ }^{9}$ ) im Einschlussrohre mit Aetzbaryt anf $100^{\circ}$ erhitzte, oder sein Hydrat, das Kreatin ${ }^{10}$ ), mit Barythydrat in wässriger Lösung kochte. Huppert ${ }^{11}$ ) und Horbaczewski ${ }^{12}$ ) erhielten dasselbe Methylbydantoïn dureh Zusammenschmelzen von Sarkosin mit Harnstoff. Endlich gewannen es E. Fischer und $\mathrm{Ach}^{13}$ ) durch Reduction des $\alpha$-Methylallantoins,

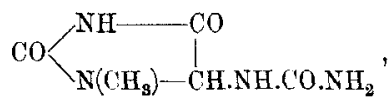

mit 60 procentiger Jodwasserstoffsäure. Bislang ist es aber durch directe Methylirung des Hydantoins noch nicht bereitet worden.

Das dritte Methylhydantoïn (III.) wurde von Franchimont und Klobbie ${ }^{14}$ ) durch directe Methylirung dargestellt. Es krystallisirt aus Alkohol in kurzen Prismen und schmilzt bei $184^{\circ}$. Seine Constitution ergiebt sich aus seinem Verhalten gegen concentrirte Salpetersäure und aus dem Verhalten seines Nitroderivates gegen heisses Wasser. Denn, wie Franchimont und Klobbie ${ }^{15}$ ) gezeigt haben, ist die Nitrirbarkeit der Ureide durch concentrirte Salpetersäure im allgemeinen auf die Anwesenheit von $\mathrm{CH}_{2}$ zurückzuführen. Dies folgt aus der Thatsache, dass die Parabansäure, die kein Methylen enthält, sich überhaupt nicht nitriren lässt und dass das Hydantoïn ein und nur ein Mononitroderivat liefert, während z. B. der Aethylenharnstoff, in welchem zwei $\mathrm{CH}_{2}$ vorhanden sind, mit Leichtigkeit in einen Dinitrokörper überführbar ist. Daraus kann man schon a priori mit grosser Wahrscheinlichkeit schliessen, dass die Nitrogruppe immer an demjenigen Stick-

\footnotetext{
9) Diese Annalen 137, 288.

10) Diese Amalen 137, 295.

11) Ber. d. deutsch. chem. Ges. 6, 1278.

1*) Monatsh. f. Chem. 8, 586.

13) Ber. d. deutsch. chem. Ges. 32, 2748.

14) Rec. trav. chim. 8, 289.

15) Ree. trav. chim. 6, 219; $7,237$.
} 
stoffatome substituirt, welches sich zwischen dem Carbonyl des Harnstoffrestes und dem Methylen des Säureradicals befindet. In dieser Annahme wird man durch den Umstand bestärkt, dass z. B. ein Hydantoïn, welches ein an diesem Stickstoffatome haftendes Metbyl enthält, nicht nitrirbar ist. Der Ort der Substitution durch $\mathrm{NO}_{2}$ lässt sich übrigens jedesmal durch Kochen des betreffenden Nitrokörpers mit Wasser experimentell erkennen.

Das unter III. angeführte Methylhydantoïn liefert beim Eindampfen mit höchst concentrirter Salpetersäure eine Nitroverbindung. Diese Thatsache allein lässt nach dem oben Gesagten ersehen, dass das dem $\mathrm{CH}_{2}$ benachbarte $\mathrm{NH}$ nicht substituirt ist, dass es vielmehr die andere Imidograppe sein muss, deren Wasserstoffatom durch Methyl ersetzt ist. Aus diesem Nitromethylhydantoïn entsteht beim Kochen mit Wasser unter Kohlensäureentwickelung Nitroamidoacetmethylamid:

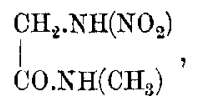

entsprechend einem Nitromethylbydantoïn von der Formel:

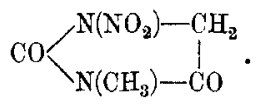

Eine von E. Fischer und $\mathrm{Ach}^{16}$ ) aufgefundene Darstellungsmethode dieses Methylhydantoïns durch Reduction des $\beta$-Methylallantoïns,<smiles>CCCCCCC(CNC(N)=O)NC(=O)O</smiles>

mittelst 60 procentiger Jodwasserstoffsäure war für seine Constitution ebenfalls beweisend und stellt also ausser allen Zweifel, dass das Methyl an dem N-Atome haftet, welches sich zwischen den beiden Carbonylen des Hydantoïnringes befindet.

Wenn wir uns bei Erörterung dieser Configurationsverhältnisse keiner auf die Constitution der verschiedenen Methyl-

16) Ber. d. deutsch. chem. Ges. 32, 2746. 
bydantoïne bezogenen Namen bedienten, so liegt das daran, dass bislang überhaupt keine consequente Nomenclatur betreffs des substituirten Hydantö̈nringes eingeführt wurde ${ }^{17}$ ). Da nun aber, wie unten $\mathrm{zu}$ besprechen ist, noch nicht beobachtete Isomerieverbältnisse eintreten können, möchten wir, nach Analogie anderer Fünfringe, die Orte der Substitution durch die Buchstaben des griechischen Alphabets nach folgendem Schema bezeichnen :
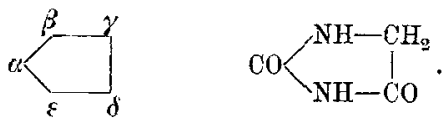

Danach behielte das unter II. angeführte, schon als $\beta$-Methylhydantoïn bezeichnete Substitutionsproduct seinen alten Namen bei, während Lactylharnstoff als $\boldsymbol{\gamma}$ - und das zweite, am Stickstoff substituirte, als $\varepsilon$-Metbylhydantoin zu bezeichnen wären.

Dieses letzte Methylhydantoïn nun ist von speciellem Interesse, weil unsere Versuche zu einem, diesem Substitionsproducte isomeren, noch nicht beobachteten Methylhydantö̈n führten. Wie bereits oben'dargethan, entsteht das Franchimont'sche $\varepsilon$-Methylhydantoïn :

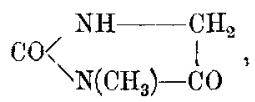

durch directe Methylirung des Kaliumsalzes des Hydantoïns in alkalischer Lösung. Wir gewannen unser neues Methylhydantoïn ebenfalls durch directe Methylirung des Hydantoïns, indem wir sein Silbersalz mit der $1 \frac{1}{4}$ fachen Menge Jodmethyl im Einschlussrohre auf $110-120^{\circ}$ erhitzten. Durch Extrahiren des neuen Productes mit Methylalkohol und Sublimiren des mehrfach umkrystallisirten Körpers erhielten wir ein Methylhydantoïn, welches constant den Schmelzp. $171^{\circ}$ zeigt, also um $13^{0}$ niedriger schmilzt, als das Franchimont'sche und das von E. Fischer und Ach durch Reduction von $\beta$-Methylallan-

17) Siehe Beilstein, Handb. d. organ. Chem., 3. Aufl, 1, 1310 und 1311. 
toin erhaltene $\varepsilon$-Methylhydantoïn. Das von Neubauer aus Kreatinin dargestellte $\beta$-Methylhydantoïn :<smiles>O=C1CCCCN1</smiles>

schmilzt bei $156^{\circ}$, also um $15^{\circ}$ tiefer, als unser Methylproduct. Indem das $\beta$-Methylhydantoïn die Möglichkeit eines Nitroderivates ausschliesst, stellt sich das neu aufgefundene, durch sein Verhalten gegen concentrirte Salpetersäure, in nahe Beziehung zum $\varepsilon$-Methylhydantoïn. Denn es liefert, wie dieses, einen Nitrokörper, der denselben Schmelzp. $168^{\circ}$ zeigt, wie das Franchimont'sche $\beta$-Nitro- $\varepsilon$-metbylhydantoin. Es ist also sehr wahrscheinlich, dass hier eine ähnliche Isomerie vorliegt, wie sie bei einzelnen monomethylirten Harnsäuren festgestellt wurde, und es besitzt dieser Isomeriefall deshalb ein erhöhtes Interesse, weil hier die Verhältnisse einfachere sind, als bei der complicirteren Harnsäure.

Bekanntlich sind durch die Anwesenheit von vier NHGruppen in der Harnsäure vier monomethylirte Harnsäuren vorauszusehen und auch bereits dargestellt. In Wirklichkeit sind aber ausser diesen noch zwei Isomere aufgefunden worden, eines von Loeben ${ }^{18}$ ), nach der synthetischen Methode von Behrend und Roosen, durch Condensation von Methylisodialursäure mit Harnstoff und ein zweites ron E. Fischer und $\mathrm{Ach}^{19}$ ) durch Methylirung des harnsauren Kaliums in schwach essigsaurer Lösung. Wie nun die letztgenannten Autoren bewiesen haben, befindet sich in ihrer $\zeta$-Methylharnsäure und in der Loeben'schen $\delta$-Methylharnsäure das Methyl in derselben Stellung wie in der Hill'schen $\alpha$-Methylharnsäure, nämlich in Stellung 3. Es kommt also allen drei substituirten Säuren die Formel :

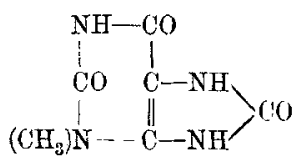

18) Diese Annalen 298, 181.

19) Ber. d. dentsch. chem. Ges. 32, 2721. 
zu. Obwohl aber die $\delta$ - und $\zeta$-Methylharnsäure und einzelne ihrer Salze durch ihre physikalischen Eigenschaften kaum zu unterscheiden sind, zeigen sie doch einen ganz charakteristischen Unterschied in ihrem Verhalten gegen Phosphoroxychlorid. Während die $\zeta$-Methylharnsäure bei $130^{\circ}$ von Phosphoroxychlorid leicht angegriffen wird und eine in diesem Agens schwer lösliche Chlorverbindung liefert, wird die $\delta$-Methylharnsäure dadurch nur schwer angegriffen und in kein schwerlösliches Chlorid übergeführt. Was nun für beide Säuren denselben ort der Substitution wahrscheinlich macht, ist der Umstand, dass beide durch Erhitzen mit Phosphoroxychlorid auf 140-1450 dasselbe 3-Methylchlorxanthin geben. Da letzteres durch Erhitzen mit Salzsäure in $\alpha$-Methylharnsäure übergeht, ist die nahe Beziehung der $\alpha-, \delta$ - und $\zeta$-Methylharnsäure festgelegt. Und zwar schlossen E. Fischer und Ach aus der Umwandlungsfähigkeit der beiden nenen Säuren in 3-Methylchlorxantbin auf den oben angedeuteten Ort der Substitution, der sich dann für die $\alpha$-Methylharnsäure von selbst ergiebt. Fischer und Ach haben es aber nicht allein bei diesem Versuche bewenden lassen, sondern diese Annahme auch auf andere Weise bekräftigt, indem sie zeigten, dass alle diese drei Methylharnsäuren durch Oxydation mit Kaliumpermanganat oder Bleisuperoxyd dasselbe Methylallantoïn liefern.

Es waren dies die ersten Fälle von Isomerie in der Harnsäuregruppe, welche nur durch Heranziehung von Lactam- und Lactim-Formen hinlänglich zu deuten waren. So hat dann E. Fischer neben der obigen, gebräuchlichen Lactamformel die folgenden vier Formeln mit einer Lactimgruppe:

I.

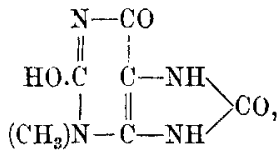

II.

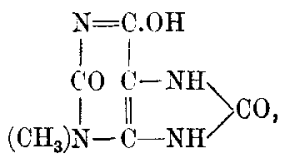


III.<smiles>Cn1c(=O)[nH]c2sc(=O)[nH]c21</smiles>

IV.<smiles></smiles>

und vier weitere mit zwei Lactimgruppen abgeleitet:<smiles></smiles>

VII.

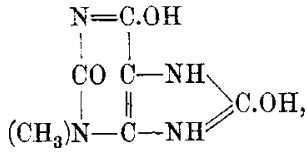

VI.<smiles>Cn1[nH]c(=O)c(O)nc1=O</smiles>

VIII.<smiles>Cn1c2nc(=O)c3c1[nH]c(=O)n3o2</smiles>

Das neue Methylhydantoïn nun weist auf die Möglichkeit ähnlicher Isomerieverhältnisse im Hydantö̈nringe hin, die ebenfalls nur durch Annahme von Lactimformen zu erklären sind. Von einer Polymerie kann auch hier nicht gut die Rede sein, wenigstens macht die ähnliche Krystallgestalt der beiden Methylhydantoine eine solche sehr unwahrscheinlich. Der Fischerschen Auffassungsweise der Stickstoffisomerie entsprechend, kommen also zu den bereits bekannten monomethylirten Hydantoïnen noch drei hinzu, und zwar:

I.

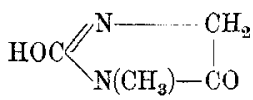

II.

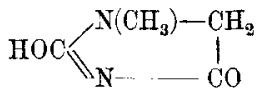

III.

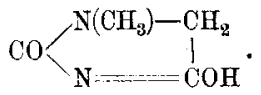

Alle drei Formeln enthalten ein saures Hydroxyl, demgemäss ist das in Rede stehende Methylhydantoïn in Alkali löslich. Doch kann demselben - gestützt auf die beim $\varepsilon$-Methylhydantoïn auseinandergesetzte Theorie der Nitroderivate die Formel II. oder III. nicht zukommen. Liefert das neue Methylhydantö̈n einen Nitrokörper, so bleibt nur Formel I. übrig, die auch die Wanderfähigkeit des Hydroxylwasserstoffs und darauffolgende Substitution durch $\mathrm{NO}_{2}$ leicht erscheinen lässt. 
Sind nun auch die den Formeln II. und III. entsprechenden Verbindungen noch nicht aufgefunden und konnten auch die etwaige Stabilität, sowie andere Eigenschaften unseres Metbylhydantoïns wegen Substanzmangels leider nicht erprobt werden, so ist dasselbe doch geeignet, die Fischer'sche Annahme der Isomerieverhältnisse $\mathrm{zu}$ bekräftigen. Namentlich gilt dies für den Fünfring in der jetzt gebräuchlichen Harnsäureformel. Betrachtet man nämlich in dieser:

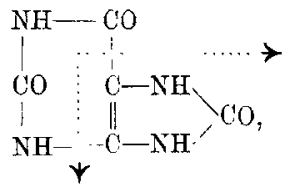

den durch die punktirte Linie abgetrennten Theil als Hydantoinring, so lässt die an diesem festgestellte Isomerie zunächst Bindungsunterschiede im Sinne der Lactimformen als möglich gelten. Andererseits lässt sie solche Isomere voraussehen, bei denen das Methyl in den NH-Gruppen dieses Ringes enthalten ist.

Durch Kochen einer Lösung von Hydantoïn in Essigsäureanhydrid erhielten wir ein bei $143-144^{\circ}$ schmelzendes Acetylderivat, welches auf Grund der Theorie der Nitroderivate als $\beta$-Monoacetylhydantoïn,

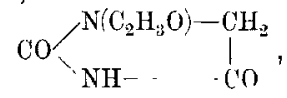

anzusprechen ist; es wird nämlich durch Salpetersäure nicht nitrirt.

Gechlorte Hydantoïne waren bislang nicht bekannt. Wir gewannen eines in Form schöner, weisser Nadeln vom Schmelzpunkt $120-121^{\circ}$ durch Einleiten von Chlor in die wässrige Lösung des Hydantoïns. Die Analyse ergab einen Gehalt von zwei At. Chlor und wir sind deshalb geneigt, demselben die Constitutionsformel

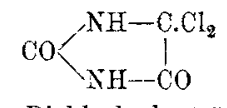

$\gamma$-Dichlorhydantoïn 
zuzusprechen. Es geschieht dies nach Analogie des Verhaltens der Barbitursäure, welche dem Hydantö̈n in der Alloxanreihe entspricht und in welcher bei der Einwirkung von Phosphoroxychlorid zwei At. $\mathrm{H}$ durch $\mathrm{Cl}_{2}$ in der $\mathrm{CH}_{2}$-Gruppe substituirt werden. Einen Beweis für diese Annahme zu erbringen, ist indessen nicht möglich gewesen. Die in dieser Richtung angestellten Versuche finden sich nebst anderen, weniger wichtigen Hydantoïnderivaten im experimentellen Theile dieser Arbeit.

\section{Experimenteller Theil.}

\section{Hydantoïnsäureester und IIydantoinn ${ }^{20}$ ).}

Wie in dem einleitenden Theile vorliegender Arbeit bereits dargelegt, diente zur Darstellung des für die Synthese des Hydantoïns angewandten Hydantoïnsäureesters das Glycocoll als das eine Ausgangsmaterial und möchten wir deshalb vorerst über die

\section{Darstellung des Glycocolläthylesterhydrochlorats}

einige Bemerkungen vorausschicken.

Steht zur Bereitung kleinerer Mengen dieses Körpers reines Glycocoll zur Verfügung, so kann man zweckmässig nach folgender Methode verfahren: Das für sich in Alkohol fast gänzlich unlösliche Glycocoll wird in fein gepulvertem Zustande in einem Erlenmeyer'schen Kolben mit absolutem Alkohol übergossen - und zwar nimmt man am besten für je $25 \mathrm{~g}$ Glycocoll $80 \mathrm{ccm}$ absoluten Alkohol --, leitet in das zum Sieden erhitzte Gemenge bis zur vollständigen Lösung, dem Endpunkte der Esterificirung, gut getrocknetes Salzsäuregas ein und bringt das syrupöse Reactionsgemisch durch Einstellen in ein Kältegemisch zum raschen Erstarren. Der aus langen, glänzenden Nadeln bestehende Krystallkuchen wird abgesaugt und mit wenig Alkohol gewaschen, sodann im Wasserbade getrocknet. Lässt

20) Harries und M. Weiss, Ber. d. dentsch. chem. Ges, B8, 3418 (1900).

Annalen der Chomie 327. Bd. 
man die Mutterlauge erstarren, was erst nach tagelangem Stehenlassen erfolgt und behandelt auch die zweite Krystallisation auf dieselbe Weise, so gestaltet sich die Ausbeute an reinem salzsauren Glycocollester zu einer fast quantitativen.

Für die Darstellung grösserer Mengen dieses Esters eignet sich das von Curtius und Goebel ${ }^{21}$ ) angegebene, von Hantzsch und Silberrad ${ }^{22}$ ) in ausgezeichneter Weise ausgebaute Verfahren. Nach dieser Methode gelangt man - von der Chloressigsäure ausgehend - auf eine sehr bequeme Weise zum Ziele, indem man die umständliche Darstellung des freien Glycocolls umgehen kann.

Das nach diesen Methoden gewonnene Glycocolläthylesterbydrochlorat kann direct zur

\section{Darstellung des Hydantö̈nsäureäthylesters}

verwandt werden.

Für dieselbe wurden, als am geeignetsten, folgende Bedingungen befunden: Molekulare Mengen salzsauren Glycocollesters und möglichst frisch bereiteten Kaliumcyanats ${ }^{23}$ ) werden in concentrirten wässrigen Lösungen rasch vermischt und bis zum Erscheinen der ersten, sich abscheidenden Krystalle heftig umgerührt, sodann in ein Kältegemisch gestellt, worauf sich der Ester als compacte Masse fest zusammenhaftender, weisser Krystallnadeln abscheidet. Bei den vorhandenen Mengen Wassers wird eine fast quantitative Trennung von Ester und Chlorkalium erzielt und man entfernt noch geringe Antheile des letzteren, indem man die auf Thonplatten getrockneten Krystalle aus vier Theilen absoluten Alkohols umkrystallisirt, aus dem die Substanz in vieleckigen, glänzenden Krystallen anschiesst. Die Ausbeute beträgt $90 \mathrm{pC}$. der Theorie.

Der Hydantoïnsäureester ist in Aether unlöslich und wird aus der Lösung in Alkohol durch Aether krystallinisch gefällt.

\footnotetext{
21) Journ. f. pract. Chem. 37, 159.

22) Ber. d. deutsch. chem. Ges. 33, 70.

23) Erdmann, Ber. d. dentsch. chem. Ges. 26, 2442.
} 
In heissem Wasser sehr leicht löslich, krystallisirt er daraus in derben Nadeln vom Schmelzp. $135^{\circ}$. Die Analyse ist bereits publicirt worden.

Es wurde das Verhalten des Esters gegen salpetrige Säure und gegen Ammoniak studirt. Während er mit ersterer einen Nitrosokörper liefert, erleidet er durch Einwirkung von Ammoniak eine unten zu besprechende Spaltung.

Der

\section{Nitrosohydantö̈nsäureäthylester}

wurde auf folgende Weise erhalten: $5 \mathrm{~g}$ Ester wurden bei gewöhnlicher Temperatur in $60 \mathrm{ccm}$ zehnprocentiger Salzsäure gelöst und mit der wässrigen Lösung der berechneten Menge $(2,36 \mathrm{~g})$ Natriumuitrit versetzt. Nachdem man das Reactionsgemisch in einer Kältemischung hat stehen lassen, scheidet sich der Nitrosokörper in Form eines leichten Schaumes an der Oberfläche der Flüssigkeit $\mathrm{ab}$. $\mathrm{Zu}$ seiner Isolirung schüttelt man mit Aether aus, lässt letzteren bei niedriger Temperatur verdunsten und krystallisirt den Nitrosokörper aus Alkohol-Aether um. Die Ausbeute betrug $0,3 \mathrm{~g}$.

Der Nitrosohydantoïnsäureäthylester, eine sehr leicht zersetzliche Verbindung, ist löslich in einem Theil heissen Alkohols, woraus er in viereckigen Tafeln krystallisirt; aus Wasser, wovon er einen halben Theil zur Lösung bedarf, schiesst er in langen Nadeln vom Schmelzp. $66-67^{\circ}$ an.

$0,2524 \mathrm{~g}$, im Vacuum getrocknet, gaben $0,3152 \mathrm{CO}_{2}$ und $0,1122 \mathrm{H}_{2} \mathrm{O}$. $0,1538 \mathrm{~g}$ gaben $31,8 \mathrm{ccm}$ Stickgas bei $20^{\circ}$ und $764 \mathrm{~mm}$ Druck.

$\begin{array}{ccr} & \text { Berechnet für } & \text { Gefunden } \\ & \mathrm{C}_{5} \mathrm{H}_{9} \mathrm{O}_{4} \mathrm{~N}_{3} & \\ \mathrm{C} & 34,28 & 34,06 \\ \mathrm{H} & 5,14 & 4,93 \\ \mathrm{~N} & 24,00 & 23,78\end{array}$

Bei der Reduction mit Aluminiumamalgam in ätherischer Lösung wird die Nitrosogruppe glatt abgespalten und es entsteht der Hydantoïnsäureäthylester znrück. 
Bei der

Einwirkung alkoholischen Ammoniaks auf den Hydantö̈nsäureäthylester

findet ganz glatt Spaltung in Glycocollamid und Harnstoff, wahrscheinlich im Sinne folgender Gleichung:

statt.

$$
\begin{aligned}
& \mathrm{CO}_{\mathrm{NH} \cdot \mathrm{CH}_{2} \cdot \mathrm{COO} \cdot \mathrm{C}_{2} \mathrm{H}_{5}}^{\mathrm{NH}_{2}}+2 \mathrm{NH}_{2}= \\
& \mathrm{CH}_{2} \cdot \mathrm{NH}_{2} \\
& \mathrm{CO} . \mathrm{NH}_{2}
\end{aligned}
$$

Nach fünfstündigem Erhitzen des Esters (5 g) mit concentrirtem alkoholischen Ammoniak (75 ccm) im Einschlussrohre auf $100^{\circ}$ scheidet sich das Glycocollamid aus der erkalteten Mutterlauge in wohlausgebildeten Krystallen aus. Dieselben wurden mit Alkohol ausgekocht und durch wiederholtes Auflösen in Wasser und darauffolgender Fällung mit Alkohol gereinigt.

$0,2644 \mathrm{~g}$, bei $100^{\circ}$ getrocknet, gaben $0,3120 \mathrm{CO}_{2}$ and $0,1898 \mathrm{H}_{2} \mathrm{O}$.

$0,1498 \mathrm{~g}$ gaben $49,8 \mathrm{ccm}$ Stickgas bei $24^{\circ}$ und $766 \mathrm{~mm}$ Druck.

$\begin{array}{ccr} & \text { Berechnet fiir } & \text { Gefunden } \\ & \mathrm{C}_{2} \mathrm{H}_{6} O \mathrm{~N}_{2} & \\ \mathrm{C} & 32,43 & 32,18 \\ \mathrm{H} & 8,11 & 7,97 \\ \mathrm{~N} & 37,83 & 37,60\end{array}$

Der nach dem Eindampfen der Mutterlauge verbleibende Rückstand besitzt den Schmelzpunkt des Harnstoffs von $130^{\circ}$ bis $132^{\circ}$, nachdem er mehrere Male aus Alkohol umkrystallisirt wurde. Seine Identität wurde auch durch die Analyse bestätigt.

$0,2700 \mathrm{~g}$ gaben $0,1962 \mathrm{CO}_{2}$ und $0,1584 \mathrm{H}_{2} \mathrm{O}$.

$\begin{array}{ccc} & \text { Berechnet für } & \text { Gefunden } \\ & \mathrm{CH}_{4} \mathrm{ON}_{2} & \\ \mathrm{C} & 20,00 & 19,82 \\ \mathrm{H} & 6,66 & 6,52\end{array}$




\section{Darstellungsweisen des Hydantö̈ns.}

\section{a. Durch Schmelzen des Hydantoünsäureesters.}

$2 \mathrm{~g}$ des Esters wurden im Schwefelsäurebade auf $135^{\circ}$ bis $140^{\circ}$ erhitzt. Im Laufe des Erhitzens scheiden sich aus der im Flusse befindlichen Masse nach und nach blättrige Krystalle $a b$, während das Reactionsgemisch eine schmutziggrüne Farbe annimmt. Als Endpunkt der Umwandlung wurde jener Moment betrachtet, in welchem beinahe die ganze Schmelze krystallinisch erstarrt war, was bei Anwendung der oben angegebenen Menge des Esters nach circa sieben Stunden der Fall war.

Nach dem Erkalten wurde in Alkohol gelöst und die grünliche Lösung durch Kochen mit Thierkohle entfärbt. Das beim Einengen der Mutterlauge sich abscheidende Product wurde aus demselben Lösungsmittel nochmals umkrystallisirt, woraus das Hydantoïn in warzenförmig angeordneten Nadeln anschiesst. Das auf diese Weise gewonnene Hydantoïn zeigt den schon bekannten Schmelzpunkt von $216^{\circ}$. Die Ausbeute betrug $0,7 \mathrm{~g}$ oder $60 \mathrm{pC}$. der Theorie, für Glycocoll berechnet $51 \mathrm{pC}$. der Theorie.

Ueberschreitet man beim Schmelzen des Hydantoïnsäureesters die genannte Temperatur, so erhält man eine schmierige Masse, die nur wenig Hydantoïn enthält.

\section{b. Durch Behandeln des Hydantoinsäureesters mit Salzsäure} gestaltet sich die Darstellungsweise des Hydantoïns zu einer eleganteren als die vorhergehende und ist derselben auch wegen besserer Ausbeute vorzuziehen.

Diese bereits von Mouneyrat ${ }^{24}$ ) bei der Darstellung substituirter Hydantoïne angewandte Methode wurde für die Bereitung des Hydantoins in mehreren Fällen erprobt und folgendes Verfabren für das beste befunden:

Von beliebigen Mengen des Hydantoïnsäureesters wird in kochender 25 procentiger Salzsäure eine fast gesättigte Lösung

24) Ber, d. deutsch. chem. Ges. B3, 2393. 
bereitet und diese verhältnissmässig kurze Zeit im Wasserbade weiter erwärmt. Es genügt z, B. 3/4 stündiges Erhitzen von $200 \mathrm{~g}$ des Esters, um seine vollständige Umwandlung in Hydantoïn zu bewirken. Lässt man nach genügend langem Erhitzen langsam erkalten, so scheidet sich die grösste Menge des Hydantoïns in schon fast reinem Zustande in Form langer Nadeln ab, die man aus Wasser nochmals umkrystallisirt. Durch Einengen der Mutterlauge und Umkrystallisiren der zweiten und auch der dritten Krystallisation aus Wasser gestaltet sich die Ausbeute an reinem Hydantoin zu einer der Theorie nabezu entsprechenden.

Auch das nach dieser Methode dargestellte Hydantoïn zeigt den Schmelzp. $216^{\circ}$ bei mittlerem Erhitzen.

Auch nach dem Eindampfen einer Lösung von einem Theil des Esters mit fünf Theilen concentrirter Salpetersäure und Umkrystallisiren des weissen Rückstandes aus Alkohol erhält man Hydantoïn vom oben angegebenen Schmelzpunkt.

Das Hydantoïn bedarf zur Lösung zwei bis drei Theile heissen Wassers und ungefähr 60 Theile siedenden Alkohols. In Aether so gut wie unlöslich, wird es aus seiner wässrigen Lösung beim Schütteln derselben mit Aether krystallinisch gefällt, scheidet sich aber aus der alkoholischen Lösung auf $\mathrm{Zu}$ satz von Aether flockig ab. Das Hydantoïn ist nicht sublimirbar.

Wie mit dem Kaliumcyanat zn Hydantoïnsäureester, setzt sich der salzsaure Glycocollester in analoger Weise mit Kaliumsulfocyanat zu dem entsprechenden Thiohydantoïnsäureester,<smiles></smiles>

um. Schon beim Zusammenbringen der alkoholischen Lösungen der beiden Agentien in der Kälte findet spontan Finwirkung statt, was sich durch die sofort erfolgende Fällung von Chlorkalium kundgiebt. Wahrscheinlich bildet sich aber zunächst der rhodanwasserstoffsaure Glycocollester, dessen Umlagerung 
in den isomeren Thiohydantoïnsäureester sich erst bei andauerndem Erhitzen seiner Lösung vollzieht. Zur

\section{Darstellung des Thiohydantoïnsäureäthylesters}

wurden concentrirte alkoholische Lösungen von $5 \mathrm{~g}$ salzsaurem Glycocollester und der berechneten Menge (ein Mol.) Rhodankalium vier bis funf Stunden in einem lebhaft siedenden Wasserbade erhitzt, nachher die vom Chlorkalium abfiltrirte Lösung im Vacuum bei $40^{\circ}$ eingedampft. Der Rückstand wurde in wenig Alkohol gelöst und durch freiwilliges Verdunstenlassen auskrystallisirt. Ausbeute 4,5 g, also $77,5 \mathrm{pC}$. der Theorie.

Der 'Thiohydantoïnsäureester ist leicht löslich in Wasser, Alkohol und Benzol; unlöslich in Aether und Petroläther, wird durch diese aus seiner alkoholischen Lösung als Oel abgeschieden. Schmelzpunkt circa $65^{0}$.

$0,2838 \mathrm{~g}$, im Vacuum getrocknet, gaben $0,3840 \mathrm{CO}_{2}$ und $0,1584 \mathrm{H}_{2} \mathrm{O}$. $0,1662 \mathrm{~g}$ gaben $24,8 \mathrm{~cm}$ Stickgas bei $20^{\circ}$ and $760 \mathrm{~mm}$ Druck.

$\begin{array}{ccr} & \text { Berechnet für } & \text { Gefunden } \\ & \mathrm{C}_{5} \mathrm{H}_{10} \mathrm{O}_{2} \mathrm{~N}_{2} \mathrm{~S} & \\ \mathrm{C} & 37,04 & 36,89 \\ \mathrm{H} & 6,17 & 6,20 \\ \mathrm{~N} & 17,28 & 17,07\end{array}$

Die Hoffnung, durch Schmelzen des Thiohydantoïnsäureesters zum normalen Thiohydantoïn,<smiles>[CH]NC(=O)NCC(=O)Cl</smiles>

zu gelangen, hat sich nicht verwirklicht. Erhitzt man nämlich den Ester im Schwefelsäurebade auf $120^{\circ}$, so tritt unter Braunfärbung langsam Zersetzung ein, die zu einem dicken, nach Mercaptan riechenden Syrup führt. Denselben erhält man auch bei längerem Verweilen des Esters im Wasserbade.

Ebensowenig ist es gelungen, durch Behandeln des Esters mit Salzsäure zum Ziele zu kommen. Salzsäure bewirkt eine die Verseifung überschreitende Spaltung, was aus der Bildung von Chlorammonium zu ersehen war. 
Auch der Versuch, durch längeres Erhitzen des Hydantoïns selbst mit Phosphortrisulfid den Thiokörper zu erhalten, zeitigte kein positives Resultat, indem auch auf diese Weise ein unerquicklicher Syrup entsteht.

Endlich wurde versucht, durch Heranziehung der von E. Fischer ${ }^{25}$ ) in der Harnsäuregruppe mit Erfolg angewandten Sulfurirungsmethode ein geschwefeltes Hydantoïn zu erhalten, es zeigte sich aber, dass bei der

\section{Einwirkung von Schwefelammonium auf Hydantö̈n}

letzteres eine Spaltung erleidet, wobei Glycocoll auftritt.

$2 \mathrm{~g}$ Hydantoïn, in $30 \mathrm{ccm}$ gelbem Schwefelammonium, wie es zu analytischen Zwecken gebraucht wird, gelöst, wurden im Einschlussrohre zwei Stunden auf $150-155^{\circ}$ erhitzt. Die nach dem Erhitzen fast farblos gewordene Lösung wurde im Wasserbade eingedampft und der weisse Rückstand - nach erfolgter Prüfung seines Verhaltens gegen Lösungsmittel - durch wiederholtes Auflösen in Wasser und darauffolgender Fällung mit dem doppelten Volum Alkohol gereinigt. So verfahren, wurde $1 \mathrm{~g}$ einer weissen Substanz gewonnen, die nicht die geringste Spur Schwefel enthält und annähernd den Schmelzpunkt des Glycocolls $\left(230-236^{\circ}\right)$ zeigt. Als solches wurde sie durch die Analyse identificirt.

$0,2121 \mathrm{~g}$, bei $100^{\circ}$ getrocknet, gaben $0,2476 \mathrm{CO}_{2}$ und $0,1234 \mathrm{H}_{2} \mathrm{O}$.

$0,1851 \mathrm{~g}$ gaben $29,4 \mathrm{~cm}$ Stickgas bei $18,5^{\circ}$ und $769 \mathrm{~mm}$ Druck.

$\begin{array}{ccc} & \text { Berechnet für } & \text { Gefunden } \\ & \mathrm{C}_{9} \mathrm{H}_{5} \mathrm{O}_{2} \mathrm{~N} & \\ \mathrm{C} & 32,00 & 31,83 \\ \mathrm{H} & 6,66 & 6,46 \\ \mathrm{~N} & 18,66 & 18,52\end{array}$

\section{Derivate des Hydantö̈ns.}

Mit dem aus dem Hydantoïnsäureester durch Behandeln mit Salzsäure in grösseren Mengen gewonnenen Hydantoïn wurden - in Aubetracht der geringen Anzahl von bekannten

25) Diese Anmalen $\mathbf{2 8 8 ,} 159$. 
Abkömmlingen - Versuche zur Darstellung von Derivaten angestellt. Bei dieser Gelegenheit hat sich die schon von anderer Seite gemachte Beobachtung der leichten Spaltbarkeit des Hydantoïnringes bestätigt. Während aber das Hydantoin schon bei der Einwirkung verbältnissmässig wenig energischer Agentien, wie z. B. von Schwefelammonium, eine Spaltung erfährt, zeigt es andererseits doch eine gewisse Resistenz, indem es z. B. sehr häufig die Neigung äussert, sich aus seinen Derivaten zurückzubilden.

Bei Besprechung der Hydantoïnderivate möchten wir zunächst über einen

\section{Versuch zur Reduction des $\beta$-Nitrohydantoüns} berichten.

Nach der von Franchimont und Klobbie ${ }^{26}$ ) angegebenen, in dieser Körperklasse ganz allgemein brauchbaren Nitrirungsmethode löst man einen Theil Hydantoïn in fünf Theilen ,höchst concentrirter Salpetersäure“ und lässt diese Lösung langsam verdunsten. Um, nach häufigen Misserfolgen, den oft gebrauchten Begriff der, ,höchst concentrirten Salpetersäure" festzulegen, wurden mehrere Nitrirungsversuche vorgenommen und es hat sich für diesen Zweck als am brauchbarsten die Salpetersäure erwiesen, welche man erhält, wenn rothe rauchende Salpetersäure in einem enghalsigen Kolben im Wasserbade so lange erhitzt wird, bis sie fast ganz farblos geworden ist. Nitrirt man mit dieser Säure, so verläuft die Reaction stets ohne Entwickelung gefürbter Dämpfe; enthält sie - wenn auch nur geringe Mengen - salpetriger Säıre, so entstehen, unter Freiwerden nitroser Dämpfe, nebenher schmierige Oxydationsproducte, die das Krystallisiren der festen Substanz erschweren.

Das nach dieser Methode dargestellte $\beta$-Nitrohydantoïn zeigt den von den Verfassern mitgetheilten Schmelzp. $170^{\circ}$.

26) Rec. trav, clim. $\boldsymbol{7}, 12$. 
Dasselbe wurde in salzsaurer Lösung mit der berechneten Menge Zinnchlorür zusammengebracht und das Reactionsgemisch nach einigem Stehen gelinde erwärmt, sodann bis zur quantitativen Fällung des Zinns mit Schwefelwasserstoffgas in der Wärme gesättigt. Der nach dem Abdampfen der filtrirten Lösung verbleibende Rückstand, aus Alkohol umkrystallisirt, zeigt den Schmelzpunkt des Hydantoins.

$0,1246 \mathrm{~g}$, bei $100^{\circ}$ getrocknet, gaben $30,2 \mathrm{ccm}$ Stickgas bei $20,5^{\circ}$ und $763 \mathrm{~mm}$ Druck.

$\begin{array}{ccc} & \text { Berechnet für } & \text { Gefunden } \\ \text { Hydantoïn } & \\ \text { N } & 28,00 & 27,45\end{array}$

Demnach findet hier keine Reduction, sondern directe $\mathrm{Ab}$ spaltung der Nitrogruppe statt.

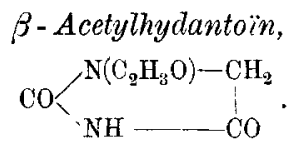

Die Constitution dieser Verbindung wurde in der Einleitung erörtert. Zu ihrer Darstellung wurden 5 g Hydantoïn mit einem Ueberschusse der zur Lösung nöthigen Menge Essigsäureanhydrids am Steigrohre drei Stunden lang erhitzt, dann aus dem etwas braun gefärbten Reactionsgemische der Acetylkörper durch Abuampfen des Lösungsmittels im Vacuum bei $50-60^{\circ}$ isolirt und aus vier Theilen Alkohol, woraus er in warzenförmig angeordneten, glänzenden Nädelchen anschiesst, umkrystallisirt. Die Ausbeute ist eine der Theorie entsprechende. Das Acetylhydantoin ist, ausser in Alkohol, auch in zwei Theilen Wasser von $100^{\circ}$ löslich, woraus es sich in derben Nadeln abscheidet; unlöslich in Aether. Schmelzp. 143-144\%

$0,2344 \mathrm{~g}$, bei $80^{\circ}$ getrocknet, gaben $0,3630 \mathrm{CO}_{2}$ und $0,0904 \mathrm{H}_{2} \mathrm{O}$.

$0,1568 \mathrm{~g}$ gaben $27 \mathrm{ecm}$ Stickgas bei $20^{\circ}$ nnd $751 \mathrm{~mm}$ Druck.

$\begin{array}{cr}\text { Berechnet für } & \text { Gefunden } \\ \mathrm{C}_{5} \mathrm{H}_{6} \mathrm{O}_{3} \mathrm{~N}_{2} & \\ 42,25 & 42,23 \\ 4,22 & 4,28 \\ 19,71 & 19,46\end{array}$


Dieses Acetylhydantoïn bleibt nach dem Abdampfen seiner Lösung in fünf Theilen höchst concentrirter Salpetersäure ganz unverändert, aus welcher Thatsache auf die oben angeführte Constitutionsformel geschlossen wurde.

Ein nicht näher untersuchtes Bleisalz des Acetylhydantoïns erhält man in büschelförmig angeordneten, glänzenden Nadeln, wenn man die mit Bleiacetat versetzte ammoniakalische Lösung des Acetylhydantoïns längere Zeit sich selbst überlässt. Durch Zusatz von Silbernitrat zur Lösung des Acetylkörpers findet keine Abscheidung eines schwer löslichen Silbersalzes statt.

$$
\text { Iso- } \varepsilon-\text { methylhydantö̈n, }
$$

Diese Verbindung ist - wie bereits erwähnt - dem von Franchimont ${ }^{27}$ ) beschriebenen Methylhydantoïn stereomer und stellt den ersten beobachteten Isomeriefall in der Klasse der einfachen Ureïde dar. Das der Bereitungsweise dieses Körpers zu Grunde liegende Silbersalz des Hydantoïns ist schon von Baeyer ${ }^{28}$ ) anlässlich der Entdeckung des Hydantoïns aufgefunden worden; er erhielt es als amorphes Pulver durch $\mathrm{Zu}$ sammenbringen von Silbernitrat mit Hydantoïn in ammoniakalischer Lösung.

Zur präparativen Darstellung grösserer Mengen dieses Salzes ist es nöthig, die Ausfällung des Silberhydantoïns in ziemlich verdünnter Lösung vorzunehmen; wendet man zu concentrirte Lösungen an, so wird durch den käsigen Niederschlag auch freies Hydantoïn mitgerissen, dessen Entfernung aus dem sehr lichtempfindlichen Silberhydantoïn mit einer nicht unbedeutenden Zersetzung des letzteren verbunden ist. Wir haben in mehreren Fällen bei Einhaltung nachstehender Arbeitsmethode sofort ein reines, fast ganz weisses Silberhydantoïn erhalten.

${ }^{27)}$ Rec. trav. chim. 8, 289.

28) Diese Annalen 180, 160. 
$\mathrm{Zu}$ einer Lösung von $17 \mathrm{~g}$ Silbernitrat in $300 \mathrm{ccm}$ Wasser liessen wir unter fortwährendem Umrühren die mit der berechneten Menge (ein Mol.) Ammoniak versetzte Lösung von $10 \mathrm{~g} \mathrm{Hydan-}$ toïn in $200 \mathrm{ccm}$ Wasser aus einem Tropftrichter langsam hinzufliessen. Hat sich der Niederschlag abgesetzt, so erwärmt man über freier Flamme, bis sich derselbe zusammengeballt hat und gut filtrirbar geworden ist, worauf man ihn, nach dem Filtriren, mit heissem Wasser und dann mit Alkohol-Aether auswäscht and unter Lichtabschluss bei $100^{\circ}$ trocknet. Das auf diese Weise in quantitativer Ausbeute gewonnene Material wurde in sehr fein zerriebenem Zustande zur Bereitung von Methylhydantoïn verwandt.

Die Methylirung selbst wurde durch Erhitzen des Silberhydantoïns mit Jodmethyl, ohne Anwendung von Methylalkohol als Lösungsmittel, bewerkstelligt. $\mathrm{Zu} 5 \mathrm{~g}$ fein zerriebenem trocknem Silberhydantoïn, welches in ein Einschlussrohr gebracht war, wurde soviel Jodmethyl hinzugefügt, bis die ganze Masse von der Flüssigkeit eben durchtränkt erscheint, wozu es ungefähr der $1^{1 / 4}$ fachen Menge Jodmethyls bedarf. Hierauf wurde während fünf Stunden auf $110-120^{\circ}$ erhitzt. Das erkaltete Reactionsgemisch, mit Methylalkohol extrahirt, giebt an das Lösungsmittel einen tiefbraun gefärbten Körper ab, der sich beim Einengen der Lösung in gelben Krusten abscheidet. Diese bestehen aus dem zu gewinnenden Methylhydantoin und zum grossen Theile aus regenerirtem Hydantoïn; sie wurden mit Methylalkohol noch einige Male gereinigt und dann, aus dem aus Hydantoïn und Methylhydantoïn bestehenden Gemenge, letzteres durch Sublimation im Sandbade bei $130-140^{\circ}$ isolirt. Das ziemlich rasch sublimirende Methylhydantoïn setzt sich wenn das Robproduct von gefärbten Verunreinigungen durch Umkrystallisiren aus Alkohol genügend befreit war - in einer Ausbeute von $6-8 \mathrm{pC}$. in schneeweissen, glänzenden Nadeln oder Flittern in reinem Zustande an.

$0,2542 \mathrm{~g}$, im Vacuum getrocknet, gaben $0,3916 \mathrm{CO}_{2}$ und $0,1152 \mathrm{H}_{2} \mathrm{O}$. $0,1462 \mathrm{~g}$ gaben $32,2 \mathrm{ccm}$ Stickgas bei $25^{\circ}$ und $762 \mathrm{~mm}$ Druck. 


$\begin{array}{ccr} & \text { Berechnet für } & \text { Gefunden } \\ & \mathrm{C}_{4} \mathrm{H}_{6} \mathrm{O}_{2} \mathrm{~N}_{2} & \\ \mathrm{C} & 42,10 & 42,00 \\ \mathrm{H} & 5,26 & 5,04 \\ \mathrm{~N} & 24,56 & 24,64\end{array}$

Das wiederholt auf diese Weise dargestellte Methylhydantoïn zeigt den Schmelzp. $171^{\circ}$, während das ihm stercomere, nach der, Alkalimethode“ von Franchimont bereitete Methylderivat bei $184^{\circ}$ schmilat. Eine Depression des Schmelzpunktes war im Gemenge der beiden Isomeren nicht zu constatiren, vielmehr zeigte dasselbe genau das arithmetische Mittel der beiden Schmelzpunkte.

Das diesem neuen Methylbydantoïn entsprechende

$$
\begin{gathered}
\beta-\text { Nitro- }- \text { - methylhydantoin, } \\
\qquad O<\begin{array}{l}
\mathrm{N}\left(\mathrm{NO}_{2}\right)-\mathrm{CH}_{3} \\
\mathrm{~N}\left(\mathrm{CH}_{3}\right)-\mathrm{CO}
\end{array}
\end{gathered}
$$

hat sich in allen seinen Eigenschaften mit dem Franchimont'schen Nitromethylhydantoïn ${ }^{29}$ ) als identisch erwiesen.

Es wurde ebenso wie dieses durch Eindampfen mit einem Theil unseres Methylhydantoïns mit fünf Theilen höchst concentrirter Salpetersäure gewonnen; hierbei findet keine Gasentwickelung, ebensowenig ein Freiwerden rother Dämpfe statt, während der Rückstand völlig weiss erscheint. Derselbe wurde aus absolutem Alkohol, in dem er ziemlich schwer löslich ist, umkrystallisirt und daraus in seideglänzenden, breiten Blättchen erhalten. Dieses Nitromethylhydantoïn zeigt denselben Schmelzpunkt wie das Franchimont'sche Product: $168^{\circ}$.

$0,1512 \mathrm{~g}$, im. Vacuum getrocknet, gaben $35,8 \mathrm{ccm}$ stickgas bei $26^{\mathrm{n}}$ und $760 \mathrm{~mm}$ Druck.

$\begin{array}{ccc} & \text { Berechnet für } & \text { Gefunden } \\ & \mathrm{C}_{4} \mathrm{H}_{5} \mathrm{O}_{4} \mathrm{~N}_{3} & \\ \mathrm{~N} & 26,41 & 26,27\end{array}$

Der Versuch, das noch unbekannte homologe Aetbylhydantoïn nach diesen beiden Methoden - der ,Alkali-“" und der

$\left.{ }^{29}\right)$ Rec. trav, chim. $\mathbf{8}, 289$. 
"Silbermethode" - darzustellen, verlief in beiden Fällen nicht mit gleichem Resultate. Während die Einwirkung von Jodmethyl in Bezug auf die Ausbeute sich bei der "Silbermethode“" als günstiger erweist, zeigt das Jodäthyl ein ganz entgegengesetztes Verhalten. Es konnte bei Anwendung der ,Alkalimethode" ein gut charakterisirtes Aethylderivat, in besserer Ausbeute als das gleiche Methylhydantoïn, gewonnen werden, keines aber durch die „Silbermethode", bei der immer freies Hydantoïn als Reactionsproduct auftrat.

An dieser Stelle sei erwähnt, dass ein Aethylhydantoïn bereits bekannt ist. Es ist von $\mathrm{Heintz}^{30}$ ) beschrieben und durch Zusammenschmelzen von Aethylglycocoll mit Harnstoff gewonnen worden. Doch hat der Autor die Constitution seiner Verbindung dahingestellt sein lassen. Da die Bildungsweise dieses Aethylhydantoïns der des $\beta$-Methylhydantoïns aus Sarkosin und Harnstoff entspricht, muss es aus den in ersten Theile vorliegender Arbeit erörterten Gründen (siehe Seite 356) als $\beta$ Aethylhydantoin,

betrachtet werden.

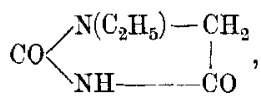

$$
\begin{gathered}
\varepsilon-\text { A thylhydantö̈n, } \\
\mathrm{CO} \\
\mathrm{NH}\left(\mathrm{C}_{2} \mathrm{H}_{5}\right)-\mathrm{CO}
\end{gathered}
$$

Diese Verbindung wurde unter Beibehaltung der von Franchimont für die Bereitung des Methylhydantoïns angegebenen Mengenverhältnisse dargestellt. Danach wurde eine Lösung ron zwei Theilen Kalilauge in 16 Theilen absoluten Alkohols mit drei Theilen Hydantoïn und sechs Theilen Jodäthyl drei bis vier Stunden auf $100--110^{\circ}$ erhitzt. Auf diese Weise erhält man ein rothbraun gefärbtes Reactionsgemisch, welches im Wasserbade zur Trockne eingedampft wird. Zur

39) Diese Annalen 133, 65 . 
Isolirung des Aethylkörpers wird mehrere Male mit absolutem Aether extrahirt und der nach dem Abdunsten des letzteren verbleibende Rückstand aus Alkohol und dann aus Wasser umkrystallisirt. Das Aethylhydantoïn krystallisirt aus Alkohol in concentrisch angeordneten, durchsichtigen Prismen, aus Wasser in rechtwinkeligen Blättchen rom Schmelzp. 1020.

$0,2500 \mathrm{~g}$, im Vacuum getrocknet, gaben $0,4286 \mathrm{CO}_{2}$ und $0,1368 \mathrm{H}_{2} \mathrm{O}$. $0,1018 \mathrm{~g}$ gaben $19,4 \mathrm{~cm}$ Stickgas bei $23^{\circ}$ und $766 \mathrm{~mm}$ Druck.

Berechnet für $\mathrm{C}_{5} \mathrm{H}_{8} \mathrm{O}_{2} \mathrm{~N}_{2}$

C

$\mathrm{H}$

N
46,87

6,25

21,88
Gefunden

46,75

6,08

21,65

Sein entsprechendes

$$
\begin{gathered}
\beta-N \text { itro- } \varepsilon-\text { Aethylhydantoin, } \\
\mathrm{CO}\left\langle\begin{array}{l}
\mathrm{N}\left(\mathrm{NO}_{2}\right)-\mathrm{CH}_{2} \\
\mathrm{~N}\left(\mathrm{C}_{2} \mathrm{H}_{5}\right)-\mathrm{CO}
\end{array}\right.
\end{gathered}
$$

wurde durch Eindampfen einer Lösung von einem Theile Aethylhydantoïn in der fünffachen Menge höchst concentrirter Salpetersäure, als ganz schwach gelb gefärbtes $0 \mathrm{el}$, das im Vacuum leicht erstarrt, erhalten. Durch Waschen mit Aether lassen sich aus dem erstarrten Abdampfungsrückstande geringe Mengen schmieriger Verunreinigungen leicht entfernen, worauf man das trockne Rohproduct aus absolutem Alkohol, in dem es nicht leicht löslich ist, umkrystallisirt. Das Nitroäthylhydantoïn schiesst aus der alkoholischen Lösung in glänzenden, dünnen Blättchen vom Schmelzp. 95-96 an.

$0,1580 \mathrm{~g}$, im Vacuum getrocknet, gaben $33,2 \mathrm{ccm}$ Stickgas bei $23^{0}$ und $768 \mathrm{~mm}$ Druck.

$\begin{array}{ccc} & \text { Berechnet fuir } & \text { Gefunden } \\ & \mathrm{C}_{5} \mathrm{H}_{7} \mathrm{O}_{4} \mathrm{~N}_{3} & \\ \mathrm{~N} & 24,27 & 23,92\end{array}$




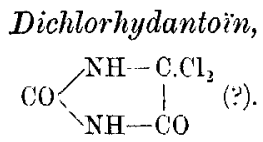

Versuche, das Hydantoïn durch Chlorphosphor in ein Chlorderivat überzuführen, waren von stetem Misserfolge begleitet. Erhitzt man nämlich Hydantoïn mit Phosphoroxychlorid im Einschlussrobre, so erfolgt schon bei $80^{\circ}$ vollständige Zersetzung, indem die ganze Masse verkohlt; diese Zerstörung tritt sogar schon beim Erhitzen bei gewöhnlichem Atmosphärendrucke ein. Nachdem sich Phosphorpentachlorid und eine Auflösung von diesem in Phosphoroxychlorid zu Chlorirungszwecken ebenfalls als unbrauchbar erwiesen haben, wurde zu einem gelinder wirkenden Mittel, dem Chlorwasser, gegriffen und mit seiner Hülfe ein gut charakterisirtes Dichlorhydantoïn gewonnen.

Zur Bereitung desselben wurden $3 \mathrm{~g}$ Hydantoïn in $50 \mathrm{ccm}$ Wasser gelöst und in diese Lösung bei einer Temperatur von $9-10^{\circ}$ und Ausschluss directen Sonnenlichts drei Stunden lang aus einer Bombe Chlor eingeleitet. In dieser Zeit haben sich aus der Lösung prächtig seideglänzende Nadeln in reinem Zustande abgeschieden, die sofort abgesaugt und mit kaltem Wasser, dann mit Aether gewaschen werden, nachher im Vacuum zu trocknen sind. Die Ausbeute beträgt $3,6 \mathrm{~g}$, also $72 \mathrm{pC}$. der Theorie.

Die in trocknem Zustande schneeweissen Krystalle zeigen den Schmelzp. $120-121^{\circ}$ und sind in Wasser und in Alkohol leicht löslich. Lässt man das Dichlorhydantoin auch nur kurze Zeit mit der chlorhaltigen Mutterlauge in Berührung, so zersetzt es sich unter Gasentwickelung.

$0,2742 \mathrm{~g}$, im Vacuum getrocknet, gaben $0,2119 \mathrm{CO}$, and $0,0248 \mathrm{H}_{2} \mathrm{O}$.

$0,1540 \mathrm{~g}$ gaben $22,2 \mathrm{~cm}$ Stickgas bei $22^{\circ}$ und $767 \mathrm{~mm}$ Druck.

$0,1922 \mathrm{~g} \quad, \quad 0,3264 \mathrm{AgCl}$.

Berechnet fuir

$$
\mathrm{C}_{3} \mathrm{H}_{2} \mathrm{~N}_{2} \mathrm{O}_{2} \mathrm{Cl}_{2}
$$

$\mathrm{C}$

$\mathrm{H}$

21,30

1,18

N

$16, \hat{\mathrm{o}} \mathrm{t}$

42,01
Gefunden

21,07

16,48

42,00 
Einen Constitutionsbeweis zu erbringen ist wegen der grossen Zersetzlichkeit dieser Verbindung in Lösungsmitteln nicht möglich gewesen. Es findet so z. B. schon beim Auflösen dieses Chlorderivates in Wasser Rückbildung von Hydantoïn statt. Von der Vermuthung geleitet, das Chlor könne in $\gamma$ Stellung getreten sein, versuchten wir, den Chlorkörper in Parabansäure überzuführen, wodurch seine Constitution bewiesen wäre. In dieser Absicht liessen wir auf das Dichlorhydantoïn in alkoholischer Lösung frisch bereitetes Silberoxyd einwirken, das isolirte Product erwies sich aber sowohl durch seinen Schmelzpunkt, als auch durch die Analyse als Hydantoïn. Den gleichen Misserfolg hatte ein Oxydationsversuch mit Salpetersäure aufzuweisen, indem hierbei Nitrohydantoïn erhalten wurde.

Ammoniak wirkt auf das Dichlorhydantoïn in wässriger and in alkoholischer Lösung unter explosionsartiger Erscheinung ein, indem sich Hydantö̈n bildet.

\section{Lacturaminsïureester und Lactylharnstoff:}

Zum Lactylharnstoff, diesem nächsten Homologen des Hydantoïns, gelangt man, indem man vom

\section{Alaninäthylesterchlorhydrat}

ausgeht und analog wie beim Hydantoin verfährt. Zur Bereitung desselben übergiesst man je $10 \mathrm{~g}$ Alanin mit $30 \mathrm{ccm}$ absolutem Alkohol und leitet unter Erwärmen bis zum Sieden wohlgetrocknetes Salzsäuregas ein, bis vollständige Lösung erfolgt, was in sebr kurzer Zeit der Fall ist. Beim Abkühlen erstarrt das Reactionsgemisch nicht, vielmehr muss es für längere Zeit in das Vacuum gebracht und häufig umgerührt werden. Nach dem Erstarren der Masse saugt man ab, wäscht zweimal mit wenig Alkohol und trocknet im Vacuum. Die Mutterlauge erstarrt im luftverdünnten Raume nochmals, wodurch die Ausbeute an reinem Alaninester zu einer der Theorie nahezu entsprechenden ergänzt werden kann. 
Die Darstellung des

$$
\begin{aligned}
& \text { Lacturaminsäureäthylesters, } \\
& \mathrm{CO} \mathrm{NH}_{3},
\end{aligned}
$$

wird bewerkstelligt durch Digestion concentrirter wässriger Lösungen von Alaninestercblorhydrat und frisch präparirtem Kaliumcyanat. Nachdem man die beiden Lösungen zusammengebracht hat, erwärmt man zweckmässig unter stetem Umrühren auf $50^{\circ}$ und bringt, sobald man die erste Abscheidung fester Substanz wahrnimmt, in ein Kältegemisch und lässt darin geraume Zeit verweilen, bis keine Abscheidung mehr erfolgt. Der so erhaltene Krystallbrei wird abgesaugt, mit wenig Wasser, nachher mit etwas Aether gewaschen und im Vacuumexsiccator getrocknet. Das trockne Rohproduct wird in Alkohol gelöst, von geringen Mengen mitgerissenen Chlorkaliums abfiltrirt und das eingeengte Filtrat zur Beschleunigung der Krystallisation mit wenig Aether versetzt. Daraus krystallisirt der Lacturaminsäureester, eine ziemlich stark hygroskopische Substanz, in langen, glänzenden Nadeln in einer Ausbeute von $76 \mathrm{pC}$. der Theorie. - Der Ester ist unlöslich in Aether, löslich in drei Theilen warmen Alkohols, in zwei Theilen Wasser, woraus er, wie aus Alkohol, in Nadeln vom Schmelzp. 93--94 krystallisirt.

$0,2564 \mathrm{~g}$, im Vacuum getrocknet, gaben $0,4214 \mathrm{CO}_{2}$ und $0,1702 \mathrm{H}_{2} \mathrm{O}$. $0,1662 \mathrm{~g}$ gaben $25 \mathrm{ccm}$ Stickgas bei $19^{\circ}$ und $762 \mathrm{~mm}$ Druck.

$\begin{array}{ccr} & \text { Berechnet fuir } & \text { Ciefunden } \\ & \mathrm{C}_{6} \mathrm{H}_{19} \mathrm{O}_{3} \mathrm{~N}_{2} & \\ \mathrm{C} & 45,00 & 44,80 \\ \mathrm{H} & 7,50 & 7,38 \\ \mathrm{~N} & 17,50 & 17,34\end{array}$

Die Alkoholabspaltung aus dem Ester wurde nach der zweckmässigeren Methode durch Behandeln mit Salzsäure bewirkt. Zwecks Bereitung des 


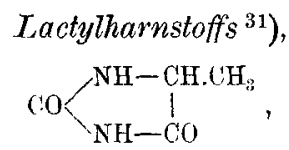

nach diesem Verfahren wurden $1,5 \mathrm{~g}$ des Lacturaminsäureesters mit $50 \mathrm{ccm} 25$ procentiger Salzsäure in Wasserbade eine Stunde lang gekocht. In Folge seines geringen Krystallisationsvermögens scheidet sich der Lactylharnstoff aus der wässrigen Mutterlauge nicht ab; es muss nach erfolgter Filtration von schleimigen Verunreinigungen zur Trockne eingedampft und der feste Rückstand aus Alkohol umkrystallisirt werden. Aus diesem Lösungsmittel schiesst der Lactylharnstoff ziemlich träge in concentrisch angeordneten Nadeln an, die schon nach dem ersten Umkrystallisiren den von Urech ${ }^{32}$ ) angegebenen Schmelzpunkt $145^{\circ}$ zeigen. Aus der angewandten Menge des Esters erhielten wir $0,9 \mathrm{~g}$ reinen Lactylharnstoff', d. i. $84 \mathrm{pC}$. der Theorie, für Alanin berechnet $65,7 \mathrm{pC}$. der Theorie.

$0,2636 \mathrm{~g}$, bei $100^{\mathrm{n}}$ getrocknet, gaben $0,4052 \mathrm{CO}_{2}$ und $0,1206 \mathrm{H}_{2} \mathrm{O}$. $0,1422 \mathrm{~g}$ gaben $30 \mathrm{ccm}$ Stickgas bei $20^{\circ}$ und $766 \mathrm{~mm}$ Drnck.

$\begin{array}{ccr} & \text { Berechnet fïr } & \text { Gefunden } \\ & \mathrm{C}_{4} \mathrm{H}_{6} \mathrm{O}_{2} \mathrm{~N}_{2} & \\ \mathrm{C} & 42,10 & 41,92 \\ \mathrm{H} & 5,26 & 5,08 \\ \mathrm{~N} & 24,56 & 24,31\end{array}$

\section{Acetyllactylharnstoff.}

Diese Verbindung wurde durch längeres Kochen von Lactylharnstoff mit einem Ueberschusse der zur Lösung nöthigen Menge Essigsäureanhydrids erhalten. Nach dem Abdestilliren des Lösungsmittels im Vacuum hinterbleibt ein gelb gefärbtes Oel, welches erst nach tagelangem Stehen im Exsiccator er-

31) Vergl. Androasch: Zur Kemitniss des Lactylharustoffs, Monatsh. f. Chemie 33, 803 (1902).

az) Diese Annalen 165, 99 und Ber. d. deutsch. chem. Ges. 6, 1113. Vergl. Heintz, diese Annalen 169,125 , der den Schmelzpunkt des Lactylharnstoff's mit $140^{\circ}$ angiebt. 
384 Harries und Weiss, Ueber das Hydantö̈n etc.

starrt. Ist dies erfolgt, so krystallisirt man das Rohproduct aus Alkohol, worin es leicht löslich ist, um; aus der alkoholischen Mutterlauge scheidet sich nach freiwilligem Verdunstenlassen des Lösungsmittels der Acetyllactylharnstoff in schönen sechsseitigen, dünnen und ganz durchsichtigen Blättchen vom Schmelzp. 129-1310 ab.

$0,2498 \mathrm{~g}$, im Vacuum getrocknet, gaben $0,4214 \mathrm{CO}_{2}$ und $0,1106 \mathrm{H}_{2} \mathrm{O}$. $0,1558 \mathrm{~g}$ gaben $24,8 \mathrm{~cm}$ Stickgas bei $24^{0}$ und $758 \mathrm{~mm}$ Druck.

$\begin{array}{ccr} & \text { Berechnet für } & \text { Gefunden } \\ & \mathrm{C}_{6} \mathrm{H}_{8} \mathrm{O}_{3} \mathrm{~N}_{2} & \\ \mathrm{C} & 46,15 & 46,00 \\ \mathrm{H} & 5,13 & 4,92 \\ \mathrm{~N} & 17,95 & 17,80\end{array}$

(Geschlossen am 9. Juni 1903.) 